\title{
ANNALES
}

$\mathrm{DE}$

\section{L'INSTITUT FOURIER}

Mathieu DUTOUR SIKIRIĆ,

Achill SCHÜRMANN \& Frank VALLENTIN

Inhomogeneous extreme forms

Tome 62, nº 6 (2012), p. 2227-2255.

<http://aif.cedram.org/item?id=AIF_2012__62_6_2227_0>

(C) Association des Annales de l'institut Fourier, 2012, tous droits réservés.

L'accès aux articles de la revue "Annales de l'institut Fourier » (http://aif.cedram.org/), implique l'accord avec les conditions générales d'utilisation (http://aif.cedram.org/legal/). Toute reproduction en tout ou partie de cet article sous quelque forme que ce soit pour tout usage autre que l'utilisation à fin strictement personnelle du copiste est constitutive d'une infraction pénale. Toute copie ou impression de ce fichier doit contenir la présente mention de copyright.

\section{cedram}

Article mis en ligne dans le cadre du

Centre de diffusion des revues académiques de mathématiques

http://www.cedram.org/ 
Ann. Inst. Fourier, Grenoble

62, 6 (2012) 2227-2255

\title{
INHOMOGENEOUS EXTREME FORMS
}

\author{
by Mathieu DUTOUR SIKIRIĆ, \\ Achill SCHÜRMANN \& Frank VALLENTIN (*)
}

\begin{abstract}
G.F. Voronoi (1868-1908) wrote two memoirs in which he describes two reduction theories for lattices, well-suited for sphere packing and covering problems. In his first memoir a characterization of locally most economic packings is given, but a corresponding result for coverings has been missing. In this paper we bridge the two classical memoirs.

By looking at the covering problem from a different perspective, we discover the missing analogue. Instead of trying to find lattices giving economical coverings we consider lattices giving, at least locally, very uneconomical ones. We classify local covering maxima up to dimension 6 and prove their existence in all dimensions beyond.

New phenomena arise: Many highly symmetric lattices turn out to give uneconomical coverings; the covering density function is not a topological Morse function. Both phenomena are in sharp contrast with the packing problem.

RÉSumÉ. - G.F. Voronoi (1868-1908) a écrit deux mémoires dans lesquels il décrit deux théories de réduction pour les réseaux, l'une adaptée aux empilements de sphères et l'autre aux recouvrements de sphères. Dans son premier mémoire une charactérisation des empilements de sphères qui sont localement les plus économiques est donnée. Dans cet article, nous relions ces deux mémoires classiques.

En considérant le problème sous un autre angle, nous faisons apparaître l'analogue manquant. Au lieu de considérer les réseaux donnant des recouvrements localement économiques, nous considérons les réseaux qui sont localement les moins économiques. Nous classifions ces réseaux jusqu'à la dimension 6 et nous prouvons leur existence dans les dimensions suivantes.

De nouveaux phénomènes apparaissent : de nombreux réseaux de haute symétrie donnent des réseaux non économiques ; la fonction de densité de recouvrement n'est pas une fonction topologique de Morse. Ces deux phénomènes sont en contraste frappant avec le cas des empilements de sphères.
\end{abstract}

Keywords: lattices, Delone polytopes, spherical $t$-designs, sphere packing, sphere covering, Voronoi reduction theory.

Math. classification: $11 \mathrm{H} 55,52 \mathrm{C} 17$.

(*) The work of the first author has been supported by the Croatian Ministry of Science, Education and Sport under contract 098-0982705-2707. The second and the third author were supported by the Deutsche Forschungsgemeinschaft (DFG) under grant SCHU 1503/4-2. The third author was supported by Vidi grant 639.032 .917 from the Dutch Organization for Scientific Research (NWO). 


\section{Introduction}

A basis of the $n$-dimensional Euclidean space $\mathbb{R}^{n}$ defines a lattice consisting of all integer linear combinations. A lattice defines a sphere packing in the following way: One centers congruent balls at the lattice points with maximum radius such that interiors do not intersect. Similarly, it defines a sphere covering: One places congruent balls with minimum radius such that each point in $\mathbb{R}^{n}$ is covered by a ball.

The (lattice sphere) packing problem asks for a lattice which gives the most economical packing, i.e., one which maximizes the fraction of space covered by the balls. The (lattice sphere) covering problem asks for a lattice which gives the most economical covering, i.e., one which minimizes the average number of balls covering a point in $\mathbb{R}^{n}$.

Many researchers were attracted by the packing problem. One important reason for this is that low-dimensional lattices which give good packings are often related to objects of exceptional beauty in combinatorics, geometry, and number theory. A vivid account of this is the monograph [9] by Conway and Sloane with over 100 pages of references which since the appearance of its first edition in 1988 spurred a tremendous amount of activity.

Our computational studies in [36], [33], [34], [18] show that the covering problem behaves very differently. Many of the best known coverings could only be discovered with computer assistance. They were found by a numerical convex continuous optimization procedure; some of them do not have a rational representation, and their beauty is not immediately apparent.

Furthermore, in [33] it came as a surprise that the root lattice $\mathrm{E}_{8}$ does not even give a locally optimal covering whereas the Leech lattice $\Lambda_{24}$ does. Both lattices are the unique optimum, up to scaling and isometries, for the lattice packing problem which was proved by Blichfeldt [4] (optimality of $\mathrm{E}_{8}$ ), Vetchinkin [38] (uniqueness of $\mathrm{E}_{8}$ ) and Cohn, Kumar [7] (optimality and uniqueness of $\Lambda_{24}$ ). In many respects both lattices behave similarly. The shortest vectors of both lattices give spherical point configurations which are optimal for many other extremal questions in geometry, like the kissing number problem and more generally for potential energy minimization which is proved in Cohn and Kumar's work on universally optimal point configurations on spheres [6].

From further experimental studies we saw that $\mathrm{E}_{8}$ is almost a local covering maximum, that is, the covering density decreases for almost all perturbations of $E_{8}$. We say that $E_{8}$ is a covering pessimum. This raised the question: Do local covering maxima exist (although local packing minima do not exist)? The first local covering maximum $\mathrm{E}_{6}$ is found in [32]. 
In this paper we develop the theory of local covering maxima. It turns out that our theory gives a new link between Voronoi's two classical memoirs [39], [40].

We think that this new theory of local covering maxima is interesting for several reasons: First of all it shows what happens to the "nice" lattices, like $\mathrm{D}_{4}, \mathrm{E}_{6}, \mathrm{E}_{7}, \mathrm{E}_{8}, \mathrm{~K}_{12}, \mathrm{BW}_{16}, \Lambda_{24}$, in the theory of lattice coverings: With the exception of the Leech lattice, all these "nice" lattices give locally very uneconomical sphere coverings. Lattices which have large covering density also come up in connection to Minkowski's conjecture. It states that every lattice $L \subseteq \mathbb{R}^{n}$ with $\operatorname{det} L=1$ satisfies

$$
\sup _{x \in \mathbb{R}^{n}} \inf _{y \in L}\left|\left(x_{1}-y_{1}\right) \cdots\left(x_{n}-y_{n}\right)\right| \leqslant 2^{-n}
$$

and equality holds only for $L=\operatorname{diag}\left(a_{1}, \ldots, a_{n}\right) \mathbb{Z}^{n}$ with $\left|a_{1} \cdots a_{n}\right|=1$. Curtis T. McMullen [25] showed that Minkowski's conjecture follows from the following covering conjecture: The (normalized) covering density of every $n$-dimensional lattice which is generated by its minimal vectors is bounded above by $\sqrt{n} / 2$ and equality holds only for lattices which are similar to the standard lattice $\mathbb{Z}^{n}$. Based on the notions developed in this paper, the second author describes an algorithm to decide the covering conjecture for every fixed dimension $n$ in [32, Chapter 5.7].

In Section 2 we start by formulating a characterization of local covering maxima in the spirit of Voronoi. In [39] Voronoi gives a similar characterization of local packing maxima extending earlier work of Korkine and Zolotarev. Then, Section 3 contains a proof of our characterization. It is based on using the Karush-Kuhn-Tucker condition from nonlinear optimization.

In Section 4 we formulate and prove a sufficient condition for being a local covering maximum in the spirit of Venkov's theory of strongly perfect lattices: It uses the $t$-design property of spherical point configurations. In [37] Venkov gives a similar condition for local packing maxima. It turns out that many interesting lattices satisfy this condition.

In Section 5 we show that there are only finitely many local covering maxima in every dimension and we give a classification which is complete up to dimension 6. For dimension 7 and 8 we give a list of all known local covering maxima. There is strong numerical evidence that these lists are complete.

One important difference between the packing problem and the covering problem is discussed in Section 6: Ash [1] proved that the packing density 
function is a topological Morse function. We show that the covering density function does not have this property if the dimension is at least four.

In the last section we give and analyze a construction showing that there are local covering maxima in all dimensions $n \geqslant 6$.

\section{Extremality $=$ Perfectness and Eutaxy}

In his first memoir Voronoi gives a characterization of locally optimal packings, building on previous works by Korkine and Zolotarev. For this he uses the notions of extremality, perfectness and eutaxy, which are naturally defined in the language of positive definite quadratic forms (PQFs).

Some preliminaries: There is a one-to-one correspondence between lattice bases up to orthogonal transformations and PQFs by taking the Gram matrix of the lattice basis. We identity the space of quadratic forms in $n$ variables with the space of real symmetric $n \times n$-matrices. It is an $\left(\begin{array}{c}n+1 \\ 2\end{array}\right)$ dimensional Euclidean space with inner product $\left\langle Q, Q^{\prime}\right\rangle=\operatorname{trace}\left(Q Q^{\prime}\right)$, where $Q$ and $Q^{\prime}$ are quadratic forms. By this identification we can evaluate a quadratic form $Q$ at a vector $x \in \mathbb{R}^{n}$ by

$$
Q[x]=x^{t} Q x=\left\langle Q, x x^{t}\right\rangle .
$$

Now we review Voronoi's characterization for the homogeneous packing case where we refer to the monographs [24] of Martinet and [32] of Schürmann for proofs and further information. Then we present our characterization for the inhomogeneous covering case.

\subsection{Homogeneous case}

Let $Q$ be a positive definite quadratic form in $n$ variables. The Hermite invariant of $Q$ is

where

$$
\gamma(Q)=\frac{\lambda(Q)}{(\operatorname{det} Q)^{1 / n}},
$$

$$
\lambda(Q)=\min _{v \in \mathbb{Z}^{n} \backslash\{0\}} Q[v],
$$

is the homogeneous minimum of $Q$. It is scale-invariant. Maximizing the packing density among lattices is equivalent to maximizing the Hermite invariant among PQFs.

Voronoi gave a characterization of the local maxima of the Hermite invariant using the geometry of the shortest vectors

$$
\operatorname{Min} Q=\left\{v \in \mathbb{Z}^{n}: Q[v]=\lambda(Q)\right\} .
$$


Definition 2.1. - Let $Q$ be a PQF.

(i) It is called extreme if it is a local maximum of the Hermite invariant.

(ii) It is called perfect if the linear space spanned by

$$
\left\{v v^{t}: v \in \operatorname{Min} Q\right\}
$$

has maximal possible rank $\left(\begin{array}{c}n+1 \\ 2\end{array}\right)$.

(iii) It is called eutactic if there are positive constants $\alpha_{v}$ so that

$$
Q^{-1}=\sum_{v \in \operatorname{Min} Q} \alpha_{v} v v^{t}
$$

It is called semieutactic if the constants are nonnegative, and weakly eutactic if the constants are real, i.e., if they exist at all.

The extended notion of semieutaxy and weak eutaxy is due to Bergé and Martinet [3]

Theorem 2.2 (Voronoi [39]). - A PQF is extreme if and only if it is perfect and eutactic.

\subsection{Inhomogeneous case}

We define the inhomogeneous Hermite invariant of a PQF $Q$ as

$$
\gamma_{i}(Q)=\frac{\mu(Q)}{(\operatorname{det} Q)^{1 / n}},
$$

where

$$
\mu(Q)=\max _{x \in \mathbb{R}^{n}} \min _{v \in \mathbb{Z}^{n}} Q[x-v]
$$

is the inhomogeneous minimum of $Q$. Like $\gamma$ it is scale-invariant. Finding extrema for the covering density among lattices is equivalent to finding extrema for the inhomogeneous Hermite invariant among PQFs.

In the literature, so far only the local minima of the inhomogeneous Hermite invariant have been considered, as they give economical coverings. However, to link the homogeneous with the inhomogeneous case we have to consider the local maxima.

In this paper we characterize local maxima of the inhomogeneous Hermite invariant using the geometry of closest vectors. For each point $c \in \mathbb{R}^{n}$ attaining $\mu(Q)$ we define the closest vectors

$$
\operatorname{Min}_{c} Q=\left\{v \in \mathbb{Z}^{n}: Q[v-c]=\mu(Q)\right\} .
$$

Geometrically, the closest vectors give the vertices of the Delone (Cyrillic: Делоне, French: Delaunay) polytope defined by the PQF $Q$ which has 
center $c$ : We have $Q[v-c]=\mu(Q)$ only for $v \in \operatorname{Min}_{c} Q$ and for all other lattice points $v \in \mathbb{Z}^{n}$ we have strict inequality $Q[v-c]>\mu(Q)$. The set of all Delone polytopes is called the Delone subdivision of $Q$ which is a $\mathbb{Z}^{n}$-periodic polyhedral subdivision of $\mathbb{R}^{n}$. The inhomogeneous minimum of $Q$ is at the same time the maximum squared circumradius of its Delone polytopes.

Definition 2.3. - Let $Q$ be a PQF.

(i) It is called inhomogeneous extreme if it is a local maximum of the inhomogeneous Hermite invariant.

(ii) It is called inhomogeneous perfect, if for each $c \in \mathbb{R}^{n}$ attaining $\mu(Q)$, the linear space spanned by

$$
\left\{\left(\begin{array}{l}
1 \\
v
\end{array}\right)\left(\begin{array}{l}
1 \\
v
\end{array}\right)^{t}: v \in \operatorname{Min}_{c} Q\right\}
$$

has maximal possible rank $\left(\begin{array}{c}n+2 \\ 2\end{array}\right)-1$.

(iii) It is called inhomogeneous eutactic, if for each $c \in \mathbb{R}^{n}$ attaining $\mu(Q)$, there are positive constants $\alpha_{v}$ so that

$$
\left(\begin{array}{cc}
1 & c^{t} \\
c & c c^{t}+\frac{\mu(Q)}{n} Q^{-1}
\end{array}\right)=\sum_{v \in \operatorname{Min}_{c} Q} \alpha_{v}\left(\begin{array}{l}
1 \\
v
\end{array}\right)\left(\begin{array}{l}
1 \\
v
\end{array}\right)^{t}
$$

It is called inhomogeneous semieutactic if the constants are nonnegative, and inhomogeneous weakly eutactic if the constants are real, i.e., if they exist.

Now we are ready to state our principal result.

TheOrem 2.4. - A PQF is inhomogeneous extreme if and only if it is inhomogeneous perfect and inhomogeneous eutactic.

We prove this theorem in Section 3 after giving a reformulation in the following subsection.

Let us contrast this characterization to the known characterization of PQFs which give local minima. Barnes and Dickson [2] gave such a characterization of PQFs in the case of generic PQF $Q$, i.e., if all Delone polytopes of $Q$ are simplices:

A generic PQF $Q$ is local minimum for $H$ if and only if one can write

$$
Q^{-1}=\sum_{c} \lambda_{c}\left(\sum_{i=0}^{n} \alpha_{i} v_{i} v_{i}^{t}-c c^{t}\right),
$$


with nonnegative $\lambda_{c}$ where the sum goes over all $c$ attaining $\mu(Q)$ and where $\operatorname{Min}_{c}(Q)=\operatorname{conv}\left\{v_{0}, \ldots, v_{n}\right\}$ and where $\alpha_{i}$ are so that $\sum_{i=0}^{n} \alpha_{i}=1$ and $c=\sum_{i=0}^{n} \alpha_{i} v_{i}$.

Hence, this characterization resembles (semi-)eutaxy; there is no perfectness here. This and the other non-generic cases where the Delone polytopes are not all simplices are discussed in [32, Chapter 5.2.4].

\subsection{Quadratic functions}

Before we go on, a remark why in the definition of inhomogeneous perfect forms the maximal possible rank is $\left(\begin{array}{c}n+2 \\ 2\end{array}\right)-1$ instead of $\left(\begin{array}{c}n+2 \\ 2\end{array}\right)$ is in order: It is $\left(\begin{array}{c}n+2 \\ 2\end{array}\right)-1$ because the vectors $v$ of $\operatorname{Min}_{c} Q$ satisfy the equation $Q[v-c]=$ $\mu(Q)$ which translates into one linear equation in the space of quadratic functions. This observation, due to Erdahl and Ryshkov [20], [22], [30], will be the key to the proof of our principal result. Let us elaborate on this.

Instead of using one quadratic form, which (implicitly) defines the inhomogeneous minimum $\mu(Q)$ and the points $c \in \mathbb{R}^{n}$ attaining $\mu(Q)$, we make things explicit by using several quadratic functions; one for each $c$. We shall explain the exact relation between a PQF and "its" quadratic functions in Section 2.4 once we have all necessary definitions.

A quadratic function in $n$ variables can be written as

$$
f(x)=\alpha_{f}+2 b_{f} \cdot x+Q_{f}[x],
$$

where $\alpha_{f} \in \mathbb{R}, b_{f} \in \mathbb{R}^{n}$, and $Q_{f}$ is a quadratic form in $n$ variables. By $b_{f} \cdot x$ we denote the standard inner product of the two $n$-dimensional vectors $b_{f}$ and $x$. We equip the space of quadratic functions with the inner product

$$
(f, g)=\alpha_{f} \alpha_{g}+2 b_{f} \cdot b_{g}+\left\langle Q_{f}, Q_{g}\right\rangle .
$$

For $x \in \mathbb{R}^{n}$ we define the quadratic function

$$
\mathrm{ev}_{x}(y)=(1+x \cdot y)^{2}
$$

which can be used to evaluate a quadratic function $f$ at $x$ by $\left(\operatorname{ev}_{x}, f\right)=$ $f(x)$. We define the Erdahl cone by

$$
\mathcal{E}_{\geqslant 0}=\left\{f: f(v) \geqslant 0 \text { for all } v \in \mathbb{Z}^{n}\right\} .
$$

If a quadratic function $f$ lies in the Erdahl cone, then $Q_{f}$ is positive semidefinite (see e.g., [20, Proposition 1.3]). We define the positive Erdahl cone by

$$
\mathcal{E}_{>0}=\left\{f \in \mathcal{E}_{\geqslant 0}: Q_{f} \text { is positive definite }\right\}
$$


Let $f$ be a quadratic function lying in the Erdahl cone. The zero set of $f$ is an ellipsoid whose interior is free of integral points, points lying in $\mathbb{Z}^{n}$. The convex hull of the integral zeroes of $f$ is called the Delone polyhedron of $f$,

$$
\text { Del } f=\operatorname{conv}\left\{v \in \mathbb{Z}^{n}: f(v)=0\right\} .
$$

Note that a Delone polyhedron might be empty, bounded or unbounded. We define the function

$$
\mu(f)=-\min _{x \in \mathbb{R}^{n}} f(x)=\max _{x \in \mathbb{R}^{n}}-f(x) .
$$

We will make extensive use of the fact that $\mu$ is a convex function. This follows because evaluation is linear in $f$. The function $\mu$ is negative exactly for those $f$ having an empty zero set so that the Delone polyhedron of $f$ is empty.

Let $f$ be a quadratic function lying in the positive Erdahl cone. If the zero set of $f$ is a non-degenerate ellipsoid (i.e., it is non-empty and bounded), then its center is $c_{f}=-Q_{f}^{-1} b_{f}$ and its squared circumradius (with respect to $\left.Q_{f}\right)$ is $\mu(f)$. In this case one can write

$$
f(x)=Q_{f}\left[x-c_{f}\right]-\mu(f), \quad \text { and } \quad \mu(f)=Q_{f}\left[c_{f}\right]-\alpha_{f} .
$$

The Hermite invariant of $f \in \mathcal{E}_{>0}$ is

$$
H(f)=\frac{\mu(f)}{\left(\operatorname{det} Q_{f}\right)^{1 / n}} .
$$

Note that it is invariant under multiplication by positive scalars.

DEFINITION 2.5. - Let $f$ be a quadratic function lying in the positive Erdahl cone.

(i) It is called extreme if it is a local maximum of the Hermite invariant.

(ii) It is called perfect, if the linear space spanned by $\mathrm{ev}_{v}$, with $v \in$ vert Del $f$, has maximal possible rank $\left(\begin{array}{c}n+2 \\ 2\end{array}\right)-1$.

(iii) It is called eutactic if there are positive real numbers $\alpha_{v}$, with $v \in$ vert $\operatorname{Del} f$, so that the following conditions hold

$$
\sum_{v \in \text { vert Del } f} \alpha_{v} \operatorname{ev}_{v}=\operatorname{ev}_{c_{f}}+\frac{\mu(f)}{n} Q_{f}^{-1} .
$$

It is called semieutactic if the constants are nonnegative, and weakly eutactic if the constants are real, i.e., if they exist.

The equation in the definition of eutaxy (iii) has the following geometric interpretation: A negative multiple of the gradient of the function $H$, which 
is given on the right hand side (see Lemma 3.2), lies in the interior of the inhomogeneous Voronoi cone

$$
\mathcal{V}(f)=\operatorname{cone}\left\{\operatorname{ev}_{x}: f(x)=0, x \in \mathbb{Z}^{n}\right\} .
$$

\subsection{Relation between quadratic forms and functions}

Let $Q$ be a PQF and $c \in \mathbb{R}^{n}$ be a point attaining the inhomogeneous minimum $\mu(Q)$. Then the closest vectors $\operatorname{Min}_{c} Q$ are the vertices of the Delone polytope Del $f$ of the quadratic function $f$ given by $Q_{f}=Q, b_{f}=$ $Q^{-1} c, \mu(f)=\mu(Q)$. Hence, the inhomogeneous minimum of $Q$ is

$$
\mu(Q)=\max \left\{\mu(f): f \text { quadratic function with } Q_{f}=Q\right\} .
$$

A side remark: The convexity of $f \mapsto \mu(f)$ immediately implies the convexity of $Q \mapsto \mu(Q)$, i.e., the main result of Delone, Dolbilin, Ryshkov, Shtogrin in [11], see also [34, Proposition 7.1] or [32, Proposition 5.1].

We can reformulate the definition of inhomogeneous perfectness and eutaxy: A PQF $Q$ is inhomogeneous perfect if all quadratic functions $f$ with $Q_{f}=Q$ and $\mu(f)=\mu(Q)$ are perfect. A PQF $Q$ is inhomogeneous eutactic if all quadratic functions $f$ with $Q_{f}=Q$ and $\mu(f)=\mu(Q)$ are eutactic. With this, Theorem 2.4 follows immediately from the following theorem.

TheOREM 2.6. - A quadratic function lying in the positive Erdahl cone is extreme if and only if it is perfect and eutactic.

\subsection{Relation to lattices}

It is well-known that there is a one-to-one correspondence between notions for PQFs (up to unimodular transformations) and notions of lattices (up to orthogonal transformations) which we briefly summarize in the following table:

\begin{tabular}{l|l} 
PQF & lattice \\
\hline determinant & volume of fundamental domain \\
homogeneous minimum & packing radius \\
Hermite invariant & packing density \\
inhomogeneous minimum & covering radius \\
inhomogenous Hermite invariant & covering density
\end{tabular}

The relation between quadratic functions and lattices is not that close. Although we use quadratic functions to describe individual Delone polytopes (and so individual vertices of the Voronoi cell of a lattice), some quadratic functions correspond to Delone polytopes, others do not. 


\section{Proof of Theorem 2.6}

The proof of our principal theorem is an analysis of local maxima of a differentiable function satisfying inequality constraints. We first recall some background from nonlinear optimization: sufficient and necessary criteria for a function to have a local maximum. Then we specialize this to our situation of the Hermite invariant of a quadratic function.

\subsection{Nonlinear optimization}

We just state the result and refer to any book on nonlinear optimization for more details, e.g., the book by Boyd and Vandenberghe [5, Chapter 5].

Let $E$ be a Euclidean space with inner product $x \cdot y$ and let $p: E \rightarrow \mathbb{R}$ and $q_{1}, \ldots, q_{k}: E \rightarrow \mathbb{R}$ be differentiable functions. Assume, we want to determine whether or not $p$ has a local maximum $x_{0}$ on the boundary of the set

$$
G=\left\{x \in E: q_{i}(x) \geqslant 0 \text { for } i=1, \ldots, k\right\} .
$$

In a sufficiently small neighborhood of $x_{0}$, the functions $p$ and $q_{i}$ can be linearized and approximated by affine functions:

$$
x \mapsto p\left(x_{0}\right)+(\operatorname{grad} p)\left(x_{0}\right) \cdot\left(x-x_{0}\right) .
$$

We define the normal cone of $G$ at $x_{0}$ by

$$
N\left(x_{0}\right)=\operatorname{cone}\left\{-\left(\operatorname{grad} q_{i}\right)\left(x_{0}\right): i=1, \ldots, k\right\} .
$$

Proposition 3.1. - Suppose $x_{0}$ satisfies $(\operatorname{grad} p)\left(x_{0}\right) \neq 0$ and $q_{i}\left(x_{0}\right)=$ 0 , as well as $\left(\operatorname{grad} q_{i}\right)\left(x_{0}\right) \neq 0$, for $i=1, \ldots, k$.

(i) The function $p$ attains an isolated local maximum on $G$ at $x_{0}$, if

$$
(\operatorname{grad} p)\left(x_{0}\right) \in \operatorname{int} N\left(x_{0}\right),
$$

where int $N\left(x_{0}\right)$ is the interior of the normal cone.

(ii) The function $p$ does not attain a local maximum on $G$ at $x_{0}$, if

$$
(\operatorname{grad} p)\left(x_{0}\right) \notin N\left(x_{0}\right) .
$$

\subsection{Proof of Theorem 2.6}

First we compute the gradient of the Hermite invariant: 
Lemma 3.2. - The Taylor series of the Hermite invariant $H$ at the quadratic function $f_{0}$ lying in the positive Erdahl cone is

$$
\frac{1}{\left(\operatorname{det} Q_{f_{0}}\right)^{1 / n}}\left(\mu\left(f_{0}\right)-\left(\operatorname{ev}_{c_{f_{0}}}+\frac{\mu\left(f_{0}\right)}{n} Q_{f_{0}}^{-1}, f-f_{0}\right)+\text { h.o.t. }\right),
$$

where h.o.t. stands for higher order terms.

Proof. - The Taylor series of the functional $\mu$ at $f_{0}$ is

$$
\mu\left(f_{0}\right)-\left(\operatorname{ev}_{c_{f_{0}}}, f-f_{0}\right)+\text { h.o.t. }
$$

and the gradient of the determinant is $(\operatorname{grad} \operatorname{det})(Q)=(\operatorname{det} Q) Q^{-1}$.

We need the following convexity result. It implies that local maxima of the Hermite invariant can only be attained at the extreme rays of the positive Erdahl cone. This and the existence of these local maxima, which we will establish in the next section, shows that the interior of the Erdahl cone is not equal to the positive Erdahl cone; although it is of course contained in it.

LEMma 3.3. - Let $f_{1}$ and $f_{2}$ be two quadratic functions in the positive Erdahl cone having positive Hermite invariants. Then, the maximum of the Hermite invariant $H$ on cone $\left\{f_{1}, f_{2}\right\}$ is only attained at its extreme rays cone $\left\{f_{1}\right\}$ or cone $\left\{f_{2}\right\}$.

Proof. - We may assume that $f_{1}$ and $f_{2}$ are not collinear. Since $H$ is scale-invariant for positive scalars we may assume that $\mu\left(f_{1}\right)=\mu\left(f_{2}\right)$. It is sufficient to prove that

$$
H\left(t f_{1}+(1-t) f_{2}\right)<t H\left(f_{1}\right)+(1-t) H\left(f_{2}\right)
$$

holds for all $0<t<1$. The convexity of the function $\mu$ and the convexity of the function $Q \mapsto(\operatorname{det} Q)^{-1 / n}$, immediately give the inequality (3.1), but only with " $\leqslant$ " instead of " $<$ ".

Since the function $Q \mapsto(\operatorname{det} Q)^{-1 / n}$ is strictly convex (originally due to Minkowski $[26, \S 8])$ we have equality in (3.1) if and only if both functions

$$
t \mapsto \mu\left(t f_{1}+(1-t) f_{2}\right), \text { and } \quad t \mapsto Q_{t f_{1}+(1-t) f_{2}}
$$

are constant for $0 \leqslant t \leqslant 1$. Suppose this is the case, then

$$
0=\mu\left(t f_{1}+(1-t) f_{2}\right)-t \mu\left(f_{1}\right)-(1-t) \mu\left(f_{2}\right)=-t(1-t) Q_{f_{1}}\left[b_{f_{1}}-b_{f_{2}}\right],
$$

and hence $b_{f_{1}}=b_{f_{2}}$. From this it follows that $\alpha_{f_{1}}=\alpha_{f_{2}}$, and hence $f_{1}$, $t f_{1}+(1-t) f_{2}$ and $f_{2}$ all coincide which contradicts the assumption. 
Note that the lemma and its proof show that the function $H$ is strictly convex on the line segment connecting $f_{1}$ and $f_{2}$ if $\mu\left(f_{1}\right)=\mu\left(f_{2}\right)$ and if $\mu$ is positive on the line segment.

Now we can finish the proof.

Proof of Theorem 2.6. - Suppose that $f_{0}$ is perfect and eutactic. Since the Hermite function is invariant with respect to positive scaling, we can work with the Erdahl cone intersected with the affine hyperplane $H_{f_{0}}$ orthogonal to $f_{0}$ and containing $f_{0}$. Consider the set

$$
G_{f_{0}}=\left\{f \in \mathcal{E}_{>0} \cap H_{f_{0}}:\left(\operatorname{ev}_{v}, f\right) \geqslant 0, v \in \operatorname{vert} \operatorname{Del} f_{0}\right\} .
$$

Since $f_{0}$ is perfect, the functions $\operatorname{ev}_{v}$, with $v \in$ vert Del $f_{0}$, span a subspace of codimension 1 in the $\left(\begin{array}{c}n+2 \\ 2\end{array}\right)$-dimensional space of quadratic functions. Hence, for a sufficiently small neighborhood $N_{f_{0}}$ of the point $f_{0}$ we have

$$
N_{f_{0}} \cap G_{f_{0}}=N_{f_{0}} \cap\left(\mathcal{E}_{>0} \cap H_{f_{0}}\right) .
$$

Since $f_{0}$ is eutactic and because of the gradient computation in Lemma 3.2 we have that $-(\operatorname{grad} H)\left(f_{0}\right)$ lies in the interior of the inhomogeneous Voronoi cone $\mathcal{V}\left(f_{0}\right)$. Here we take the interior within the affine hyperplane $H_{f_{0}}$. Applying Proposition 3.1 (i) shows that $f_{0}$ is a local maximum of $H$.

Conversely, suppose that $f_{0}$ is extreme. Then by Lemma 3.3 we know that $f_{0}$ has to lie on an extreme ray of the Erdahl cone, hence it is perfect. Suppose that $f_{0}$ is not eutactic. Proposition 3.1 (ii) shows that the only situation which can occur is that $-(\operatorname{grad} H)\left(f_{0}\right)$ lies on the boundary of the inhomogeneous Voronoi cone $\mathcal{V}\left(f_{0}\right)$. Then, by Farkas' lemma (see e.g., Schrijver [31, Chapter 7.3]), there exists a quadratic function $h$ in the affine hyperplane $H_{f_{0}}$ orthogonal to $f_{0}$ and containing $f_{0}$ so that

$$
\left\{\begin{array}{l}
\left(\operatorname{ev}_{v}, h\right) \geqslant 0, \quad \text { for all } v \in \operatorname{vert} \operatorname{Del} f_{0}, \\
\left((\operatorname{grad} H)\left(f_{0}\right), h\right)=0
\end{array}\right.
$$

For $\lambda \geqslant 0$, consider the univariate function

$$
\varphi_{\alpha}(\lambda)=\mu\left(f_{0}+\lambda\left(h+\alpha f_{0}\right)\right) .
$$

We can choose $\alpha$ so that

$$
0=\frac{\partial \varphi_{\alpha}}{\partial \lambda}(0)=\left((\operatorname{grad} \mu)\left(f_{0}\right), h+\alpha f_{0}\right)
$$

because $\left((\operatorname{grad} \mu)\left(f_{0}\right), f_{0}\right)=\mu\left(f_{0}\right) \neq 0$. Since $\varphi_{\alpha}$ is convex and because $\frac{\partial \varphi_{\alpha}}{\partial \lambda}(0)=0$, we have

$$
\varphi_{\alpha}(\lambda) \geqslant \varphi_{\alpha}(0)
$$


For $\lambda \geqslant 0$ consider the univariate function

$$
\psi_{\alpha}(\lambda)=\operatorname{det}\left(Q_{f_{0}}+\lambda\left(Q_{h}+\alpha Q_{f_{0}}\right)\right)^{-1 / n} .
$$

Since $\psi_{\alpha}$ is strictly convex, we have for $\lambda>0$

$$
\psi_{\alpha}(\lambda)>\psi_{\alpha}(0)+\frac{\partial \psi_{\alpha}}{\partial \lambda}(0) \lambda .
$$

Taking the product shows

$$
H\left(f_{0}+\lambda\left(h+\alpha f_{0}\right)\right)=\varphi_{\alpha}(\lambda) \psi_{\alpha}(\lambda)>\varphi_{\alpha}(0) \psi_{\alpha}(0)=H\left(f_{0}\right),
$$

because $\frac{\partial \psi_{\alpha}}{\partial \lambda}(0) \geqslant 0$. Hence, $f_{0}$ is not extreme.

\section{Examples - Strongly inhomogeneous perfect forms}

Venkov introduced strongly perfect forms in [37]. Strongly perfect forms are PQFs in which the shortest vectors carry a spherical 4-design.

TheOREM 4.1 (Venkov [37]). — Strongly perfect forms are extreme.

The notion of spherical designs is due to Delsarte, Goethals, Seidel [12]. Generally, finitely many points $X$ in $\mathbb{R}^{n}$ carry a spherical $t$-design (with respect to a $\mathrm{PQF} Q$ ) if they lie on a sphere

$$
S_{Q}(c, r)=\left\{x \in \mathbb{R}^{n}: Q[x-c]=r^{2}\right\}, \quad \text { with } c \in \mathbb{R}^{n}, \text { and } r \in \mathbb{R},
$$

and so that for all polynomials $f$ up to degree $t$ we have

$$
\frac{1}{|X|} \sum_{x \in X} f(x)=\int_{S_{Q}(c, r)} f(x) d \omega(x),
$$

where $\omega$ is the normalized surface measure on $S_{Q}(c, r)$. The maximal $t$ for which $X$ carries a spherical $t$-design is called its strength which we denote by $s(X)$. An equivalent, alternative characterization of spherical $t$-designs is the following: The points $X$ carry a spherical $t$-design (with respect to a PQF $Q$ ) if there exists $c \in \mathbb{R}^{n}$ and $r \in \mathbb{R}$ so that the following equalities hold for all $k \leqslant t$ and all $y \in \mathbb{R}^{n}$ :

$$
\sum_{x \in X}\left\langle Q,(x-c)(y-c)^{t}\right\rangle^{k}=\left\{\begin{array}{c}
0, \quad \text { for all odd } k \\
\frac{1 \cdot 3 \cdots(k-1)}{n(n+2) \cdots(n+k-2)}|X| r^{k / 2} Q[y-c]^{k / 2} \\
\quad \text { for all even } k .
\end{array}\right.
$$

For the proof of Theorem 4.1 Venkov used Voronoi's characterization of extreme PQFs in Theorem 2.2. He shows that having a spherical 2-design already implies eutaxy, and having a spherical 4-design implies perfectness. 
Theorem 4.1 gives a uniform way for showing that many remarkable PQFs are extreme. It applies e.g., to the forms of the root lattices $\mathrm{D}_{4}$, $\mathrm{E}_{6}, \mathrm{E}_{7}, \mathrm{E}_{8}$, the Coxeter-Todd lattice $\mathrm{K}_{12}$, the Barnes-Wall lattices $\mathrm{BW}_{2^{d}}$, with $d \geqslant 3$, the laminated lattice $\Lambda_{23}$, the shorter Leech lattice $\mathrm{O}_{23}$, the Leech lattice $\Lambda_{24}$, the Thompson-Smith lattice $\Lambda_{248}$. All but the last case are treated in Venkov [37]. The result that the Barnes-Wall lattices are strongly perfect is due to Nottebaum [29]. For the Thompson-Smith lattice see Lempken, Schröder, Tiep [23]. In the last two cases it is interesting to note that one can show the strong perfectness of $\mathrm{BW}_{2^{d}}$ and $\Lambda_{248}$ without having the list of all minimal vectors (in fact at the time of writing not even the inhomogeneous minimum is known) but using properties of the automorphism group of $\mathrm{BW}_{2^{d}}$ and $\Lambda_{248}$ only.

Now we adapt the concept of strong perfection to the inhomogeneous case.

DefInItION 4.2. - Let $Q$ be a PQF. It is called strongly inhomogeneous perfect, if for each $c \in \mathbb{R}^{n}$ attaining $\mu(Q)$, the closest vectors $\operatorname{Min}_{c} Q$ carry a spherical 4-design.

THEOREM 4.3. - Inhomogeneous strongly perfect forms are inhomogeneous extreme.

We also adapt the definitions to the setting of quadratic functions.

DEFINITION 4.4. - Let $f$ be a quadratic function lying in the positive Erdahl cone. It is called strongly perfect, if the vertices of its Delone polytope carry a spherical 4-design.

THEOREM 4.5. - Strongly perfect quadratic functions are extreme.

Like previously, Theorem 4.3 immediately follows from Theorem 4.5. The proof of the second theorem uses our characterization of inhomogeneous extreme forms in Theorem 2.2. It shows, like in the homogeneous case, that having spherical 2-designs already implies eutaxy, and that having spherical 4-designs implies perfectness.

Proof of Theorem 4.5. - Let $f$ be a strongly perfect quadratic function. The set $X=$ vertDel $f$ carries a spherical 4 -design with respect to the quadratic form $Q_{f}$. 
We shall show that $f$ is eutactic: If we unfold the equation in the definition of eutactic quadratic functions, we get

$$
\left\{\begin{aligned}
1 & =\sum_{x \in X} \alpha_{x}, \\
0 & =\sum_{x \in X} \alpha_{x}\left(x-c_{f}\right), \\
\frac{\mu(f)}{n} Q_{f}^{-1} & =\sum_{x \in X} \alpha_{x}\left(x-c_{f}\right)\left(x-c_{f}\right)^{t} .
\end{aligned}\right.
$$

We set $\alpha_{x}=\frac{1}{|X|}$ with $x \in X$, so that the first condition in Definition 2.5 (iii) is satisfied. Then, by looking at the alternative definition of spherical 1- and 2-designs, we see that the other two conditions are satisfied, see e.g., [33, Lemma 5.1].

We shall show that $f$ is perfect: Let $g$ be a quadratic function which satisfies the linear equations

$$
\left(\mathrm{ev}_{x}, g\right)=g(x)=0 \quad \text { for all } x \in X .
$$

Since $X$ carries a spherical 4-design, we have

$$
0=\frac{1}{|X|} \sum_{x \in X} g(x)^{2}=\int_{S_{Q_{f}}\left(c_{f}, \sqrt{\mu(f)}\right)} g(x)^{2} d \omega(x) .
$$

So, $g$ vanishes on $S_{Q_{f}}\left(c_{f}, \sqrt{\mu(f)}\right)=\left\{x \in \mathbb{R}^{n}: f(x)=0\right\}$. Hence, it has to be a multiple of $f$. So the space spanned by the functions $e_{x}$, with $x \in X$, has codimension 1 in the $\left(\begin{array}{c}n+2 \\ 2\end{array}\right)$-dimensional space of quadratic functions. In other words, $f$ is perfect.

Using Theorem 4.5 one can show that the PQFs belonging to the lattices $\mathrm{E}_{6}, \mathrm{E}_{7}, \mathrm{BW}_{16}, \Lambda_{23}, \mathrm{O}_{23}$ are inhomogeneous strongly perfect and hence inhomogeneous extreme. Geometrically this says that these lattices yield local covering maxima. These are all inhomogeneous strongly PQFs we know of. In Table 4.1 we give some details about these PQFs and the Delone polytopes: The second column gives the number of orbits of Delone polytopes. In all these cases there is only one orbit corresponding to points $c$ where $\mu(Q)$ is attained. In the last column we give a reference where a description of the orbits can be found.

The PQFs belonging to the lattices $\mathbb{Z}^{n}, \mathrm{D}_{n}, \mathrm{E}_{6}^{*}, \mathrm{E}_{7}^{*}, \mathrm{E}_{8}, \mathrm{~K}_{12}$ are not inhomogeneous perfect. However they are inhomogeneous eutactic. We will get a geometrical interpretation from Theorem 6.1: These lattices yield local covering pessima, i.e., the set of perturbations in which the covering density decreases has measure zero. Section 6 is concerned with covering pessima. In Table 4.2 we give some details about these PQFs and the Delone polytopes. 
A PQF belonging to the Leech lattice is neither inhomogeneous perfect nor inhomogeneous eutactic. In fact, geometrically, the Leech lattice gives a local minimum for the covering density, see [33].

\begin{tabular}{|c|c|c|c|c|}
\hline name & \# orbits & $\left|\operatorname{Min}_{c}(Q)\right|$ & $s\left(\operatorname{Min}_{c}(Q)\right)$ & reference \\
\hline $\mathrm{E}_{6}$ & 1 & 27 & 4 & Conway, Sloane [8] \\
\hline $\mathrm{E}_{7}$ & 2 & 56 & 5 & CS [8] \\
\hline $\mathrm{BW}_{16}$ & 4 & 512 & 5 & Dutour Sikirić, \\
\hline $\mathrm{O}_{23}$ & 5 & 94208 & 7 & $\begin{array}{l}\text { Schürmann, Vallentin [19] } \\
\text { DSV [19] }\end{array}$ \\
\hline$\Lambda_{23}$ & 709 & 47104 & 7 & $\mathrm{DSV}[19]$ \\
\hline
\end{tabular}

Table 4.1. Lattices belonging to inhomogeneous strongly perfect forms.

\begin{tabular}{c|c|c|c|l} 
name & \# orbits & $\left|\operatorname{Min}_{c}(Q)\right|$ & $s\left(\operatorname{Min}_{c}(Q)\right)$ & reference \\
\hline $\mathbb{Z}^{n}$ & 1 & $2^{n}$ & 3 & Conway, Sloane [8] \\
$\mathrm{D}_{3}$ & 2 & 6 & 3 & $\mathrm{CS}[8]$ \\
$\mathrm{D}_{4}$ & 1 & 8 & 3 & $\mathrm{CS}[8]$ \\
$\mathrm{D}_{n}, n \geqslant 5$ & 2 & $2^{n-1}$ & 3 & $\mathrm{CS}[8]$ \\
$\mathrm{E}_{6}^{*}$ & 1 & 9 & 2 & $\mathrm{CS}[8]$ \\
$\mathrm{E}_{7}^{*}$ & 1 & 16 & 3 & $\mathrm{CS}[8]$ \\
$\mathrm{E}_{8}$ & 2 & 16 & 3 & CS [8] \\
$\mathrm{K}_{12}$ & 4 & 81 & 3 & Dutour Sikirić, \\
& & & & Schürmann, Vallentin [19]
\end{tabular}

Table 4.2. Lattices belonging to inhomogeneous eutactic forms.

We finish this section by posing several problems:

(i) Are there strongly perfect functions which do not define inhomogeneous strongly perfect forms?

(ii) Is a PQF of the Barnes-Wall lattice $\mathrm{BW}_{2^{d}}$ for $d \geqslant 5$ inhomogeneous strongly perfect?

(iii) Is a PQF of the Thompson-Smith lattice $\Lambda_{248}$ inhomogeneous strongly perfect?

(iv) It would be interesting to classify strongly perfect quadratic functions in low dimensions. So far only a classification up to dimension 6 is known. It is described in the next section. In the homogeneous case, strongly perfect forms have been classified up to dimension 12 by Nebe and Venkov [28]. 


\section{Finiteness and classification}

In this section we show that there are only finitely many inequivalent perfect quadratic functions, respectively eutactic quadratic functions, in a given dimension. Here, equivalence is defined using scaling and using the action of the affine general linear group

$\operatorname{AGL}_{n}(\mathbb{Z})=\left\{u: \mathbb{R}^{n} \rightarrow \mathbb{R}^{n}: u(x)=v+A x\right.$, with $v \in \mathbb{Z}^{n}$ and $\left.A \in \mathrm{GL}_{n}(\mathbb{Z})\right\}$.

More precisely, we say that two quadratic functions $f$ and $g$ are equivalent if there exists a positive scalar $\lambda$ and $u \in \mathrm{AGL}_{n}(\mathbb{Z})$ so that $f(x)=\lambda g(u(x))$.

THEOREM 5.1. - In any dimension there are only finitely many inequivalent perfect quadratic functions, respectively weakly eutactic quadratic functions.

Proof. — From the work of Voronoi [40, §98] (see also Deza, Laurent [13, Chapter 13.3]) it follows that, up to $\mathrm{AGL}_{n}(\mathbb{Z})$ equivalence, there are only finitely many Delone polytopes of quadratic functions. This implies that there are only finitely many inequivalent perfect quadratic functions.

Now we argue that every Delone polytope $D$ determines up to equivalence at most one eutactic quadratic function. For this we define the cone

$$
\Delta(D)=\left\{f \in \mathcal{E}_{>0}: \operatorname{Del} f=D\right\} .
$$

Since the function $\mu$ is strictly positive on it, Lemma 3.3 and its proof show that $H$ has at most one critical point, which is a minimum of $H$.

If $f$ is weakly eutactic, then for all $g \in \Delta(D)$ we have

$$
\begin{aligned}
(-(\operatorname{grad} H)(f), g) & =\frac{1}{\left(\operatorname{det} Q_{f}\right)^{1 / n}}\left(\operatorname{ev}_{c_{f}}+\frac{\mu(f)}{n} Q_{f}^{-1}, g\right) \\
& =\frac{1}{\left(\operatorname{det} Q_{f}\right)^{1 / n}}\left(\sum_{v \in \text { vert Del } f} \alpha_{v} \operatorname{ev}_{v}, g\right) \\
& =0,
\end{aligned}
$$

and hence $f$ is a critical point of $H$.

Perfect quadratic functions have been classified up to dimension 6 ; the known lists in dimension 7 and 8 seem to be complete:

Dimension 2, .., 5: Erdahl [20, Theorem 5.1] showed that there are no perfect quadratic functions in dimension $n=2, \ldots, 5$.

Dimension 6: Dutour [15] showed that up to equivalence there is exactly one perfect quadratic function in dimension 6 : It is defined by the Schläfli polytope $2_{21}$ in dimension 6 having 27 vertices (see 
e.g., [10, Chapter 11.8]). It is strongly perfect since the vertices of $2_{21}$ carry a spherical 4-design.

Dimension 7: In dimension 7 there are two perfect quadratic functions known. The list is given in Dutour, Erdahl, Rybnikov [17, Section 7]: One is defined by the Gosset polytope $3_{21}$ in dimension 7 having 56 vertices (see e.g., [10, Chapter 11.8]). It is strongly perfect since the vertices of $3_{21}$ carry a spherical 5 -design. The other one is defined by the 35-tope constructed by Erdahl, Rybnikov [21]. It is eutactic, but it is not strongly perfect (the strength of the design is 0$)$.

Dimension 8: In dimension 8 there are 27 perfect quadratic functions known. They are described in Dutour, Erdahl, Rybnikov [17, Section 8]. 21 of them are eutactic, among them there is no strongly perfect quadratic function.

It would be interesting to understand the asymptotics of the number of perfect quadratic functions and the number of eutactic quadratic functions. At the moment it is not even clear whether the number grows with every dimension. This appears to be extremely likely: In dimension 9 we found more than 100, 000 perfect quadratic functions.

\section{Pessima and topological Morse functions}

In this section we study inhomogeneous eutactic forms. First we consider inhomogeneous eutactic forms which are not inhomogeneous perfect. They can be almost local maxima for the inhomogeneous Hermite invariant. By this we mean the following: A PQF is called a pessimum, if it is not a local maximum of the inhomogeneous Hermite invariant, but for which almost all local perturbations decrease it. Note that there does not exist an analogue of pessima for the homogeneous Hermite invariant: There is no PQF for which almost all local perturbations increase the Hermite invariant. However, it is known ( S̆togrin [35]) that when a PQF is eutactic then the Hermite invariant decreases in almost every direction.

TheOREM 6.1. - Let $Q$ be an inhomogeneous eutactic PQF which is not inhomogeneous extreme. Suppose for all quadratic functions $f$ lying in the positive Erdahl cone with $Q=Q_{f}$ and $\mu(Q)=\mu(f)$, the Delone polyhedron Del $f$ is not a simplex. Then $Q$ is a pessimum.

Proof. - Let $Q^{\prime}$ be a generic perturbation of $Q$ so that all Delone polytopes of $Q^{\prime}$ are simplices. Let $\Delta$ be a Delone simplex contained in a Delone 
polytope $D=\operatorname{Del} f$ of $Q$. Let $f^{\prime}$ be the quadratic function with Del $f=\Delta$ and $Q_{f^{\prime}}=Q^{\prime}$. Then we have the expansion

$$
H\left(f^{\prime}\right)=H(f)-\sum_{v \in \operatorname{vert} D} \alpha_{v}\left(f^{\prime}-f\right)(v)+\text { h.o.t. },
$$

because $f$ is eutactic. Since $D$ is not a simplex, there is a $v \in \operatorname{vert} D$ so that $\left(f^{\prime}-f\right)(v)>0$. This implies that the second summand of the expansion is negative.

This situation occurs for instance for the PQFs belonging to lattices in Table 4.2.

As a second application we show that the inhomogeneous Hermite invariant is generally not a topological Morse function. We recall the following definition from Morse [27].

Definition 6.2. - Let $M$ be an $m$-dimensional topological manifold and let $f$ be a real valued continuous function on $M$.

(i) A point $q \in M$ is called topologically ordinary if there exist neighborhoods $U$ of $q$ and $V$ of $0 \in \mathbb{R}^{m}$ and a homeomorphism $\phi: V \rightarrow U$ such that for all $x \in V$

$$
\phi(0)=q, \quad f(\phi(x))=x_{1}+f(q) .
$$

Otherwise, it is called topologically critical.

(ii) A topologically critical point is called topologically non-degenerate of index $r$ if there exist $U, V, \phi$ as above such that for all $x \in V$

$\phi(0)=q, \quad f(\phi(x))=-x_{1}^{2}-\cdots-x_{r}^{2}+x_{r+1}^{2}+\cdots+x_{m}^{2}+f(q)$.

(iii) A function is called topological Morse function if all points are either ordinary or topologically non-degenerate.

Note that at a topological non-degenerate point the directions of decrease are homotopically equivalent to the sphere $S^{r-1}=\left\{x \in \mathbb{R}^{r}:\|x\|=1\right\}$. The directions of increase are homotopically equivalent to the sphere $S^{m-r-1}$.

Since $H$ is scale invariant, it is not a topological Morse function for trivial reasons; the same is true for the homogeneous Hermite invariant $\gamma$. Ash [1] showed that $\gamma$ is a topological Morse function on the cone of positive semidefinite $n \times n$-matrices where we $\bmod$ out by positive scaling: $\mathcal{S}_{>0}^{n} / \mathbb{R}_{>0}$. As the following theorem shows, this is in general not the case for $H$.

TheOREM 6.3. - The inhomogeneous Hermite invariant is a topological Morse function on $\mathcal{S}_{>0}^{n} / \mathbb{R}_{>0}$ if and only if $n$ is at most three.

We need the following lemma: 
Lemma 6.4. - Let $Q$ be an inhomogeneous eutactic form. Then $Q$ is a topologically critical point for $H$ in $\mathcal{S}_{>0}^{n} / \mathbb{R}_{>0}$. It is a topologically nondegenerate point if and only if there exist one Delone polytope $D$ attaining the maximum circumradius such that for all Delone polytopes $D^{\prime}$ attaining the maximum circumradius we have

$$
\operatorname{lin} \Delta\left(D^{\prime}\right) \subseteq \operatorname{lin} \Delta(D),
$$

where $\Delta$ was defined in (5.1).

Proof. - Let $D_{1}, \ldots, D_{r}$ be the translation classes of Delone polytopes attaining the maximum circumradius. The argument in the proof of Theorem 6.1 shows that $H$ increases in the direction of

$$
U=\bigcup_{i=1}^{r} \operatorname{lin} \Delta\left(D_{i}\right) / \mathbb{R}_{>0} .
$$

It decreases in all other directions. So it is a topologically critical point. If $U=\operatorname{lin} \Delta\left(D_{i}\right)$ for some $D_{i}$, then $Q$ is a topologically non-degenerate point. If $U$ is a union of subspaces which is not contained in $\operatorname{lin} \Delta\left(D_{i}\right)$ for one $D_{i}$, then $U$ is not homotopically equivalent to a sphere, so $Q$ is not a topologically non-degenerate point.

Proof of Theorem 6.3. - There is at most one critical point in the secondary cone of a fixed Delone decomposition up to the action of $\mathrm{GL}_{n}(\mathbb{Z})$.

If $n$ equals two, there are two critical points: The PQF corresponding to the lattice $\mathbb{Z}^{2}$ and the one corresponding to the lattice $A_{2}$. They are both inhomogeneous eutactic. In both cases there is only one Delone polytope up to translations and antipodality. So both PQFs are topologically nondegenerate by the previous lemma.

If $n$ equals three, there are five types of Delone subdivisions (due to the Russian crystallographer E.S. Fedorov, see also Vallentin [36]). In all but the generic case one can check the following facts by inspection and elementary hand calculation: For every Delone subdivision which is not a triangulation there is a inhomogeneous eutactic PQF in which the Delone polytopes attaining the maximum circumradius are equivalent up to translations and antipodality. So we can apply the previous lemma, showing that these four points are topologically non-degenerate. In the generic case, where the subdivision is a triangulation, there is a PQF (associated to the lattice $\mathrm{A}_{3}^{*}$ ) where $H$ attains a local minimum.

If $n$ equals four, we consider the PQF which corresponds to the root lattice $\mathrm{D}_{4}$. It is inhomogeneous eutactic. There are three translation classes of Delone polytopes $D_{1}, D_{2}, D_{3}$ which are all regular cross polytopes realizing the circumradius. Their linear subspaces lin $\Delta\left(D_{i}\right)$ are not contained 
in each other, so by the preceding lemma the PQF is not topologically non-degenerate.

For $n$ greater than four, we take the PQF which corresponds to the lattice $\mathrm{D}_{4} \times \mathbb{Z}^{n-4}$.

\section{An infinite series of inhomogeneous extreme forms}

In this section we construct a series of inhomogeneous extreme forms for dimensions $n \geqslant 6$. The first two PQFs in the series correspond to the lattices $E_{6}$ and $E_{7}$. These PQFs were originally introduced in [16].

For giving the construction and for its analysis it is convenient not to work with the standard lattice but with the lattice $L_{n}$ which is spanned by the root lattice $\left(\mathrm{D}_{n-1}, 0\right)$ and the vector $\left(-1 / 2,(1 / 2)^{n-2}, 1\right)$. It comes with the PQF

$$
Q_{n}[x]= \begin{cases}x_{1}^{2}+\cdots+x_{n-1}^{2}+(n-3) / 4 x_{n}^{2} & \text { if } n \text { even } \\ x_{1}^{2}+\cdots+x_{n-1}^{2}+(n-5) / 4 x_{n}^{2} & \text { if } n \text { odd }\end{cases}
$$

We denote this pair by $\left[L_{n}, Q_{n}\right]$. We have $\left|\operatorname{Aut}\left(\left[L_{n}, Q_{n}\right]\right)\right|=\left|\operatorname{Aut}\left(\mathrm{D}_{n-1}\right)\right|$.

Theorem 7.1. - For $n \geqslant 6$, the lattice $\left[L_{n}, Q_{n}\right]$ are local covering maxima.

The main step of the computation is to prove that the big Delone polytope $P_{n}$ defined in the next section is the only one attaining the maximum circumradius. In order to show this we enumerate all Delone polytopes up to symmetry. We shall prove that our list is complete by a volume argument.

In the remaining part of this section will be used to give a proof of the theorem which is largely computational. The idea of the proof is based on the algorithms given in [19] which are implemented in [14].

In the proof we heavily rely on the computation of volumes of polyhedra: Let $P$ be a non-necessarily full dimensional polytope of $\mathbb{R}^{n} \cdot \operatorname{By} \operatorname{vol}(P)$ we denote the volume of $P$ for the volume form induced by the scalar product on the affine space aff $(P)$ defined by $P$. If $v \notin$ aff $(P)$, we will then have the relation

$$
\operatorname{vol}(\operatorname{conv}(P, v))=\frac{1}{\operatorname{dim}(\operatorname{conv}(P, v))} \operatorname{dist}(v, \operatorname{aff}(P)) \operatorname{vol}(P),
$$

where $\operatorname{conv}(P, v)$ denotes the convex hull of the polytope $P$ and the point $v$, and where $\operatorname{dist}(v, \operatorname{aff}(P))$ denotes the Euclidean distance between $v$ and $\operatorname{aff}(P)$. An easy consequence of this formula is that if aff $(P)$ is a hyperplane 
of dimension $n-1$ defined by an affine equality $\phi(x)=0$, then we have for $v, v^{\prime} \notin \operatorname{aff}(P)$ the relation

$$
\operatorname{vol}(\operatorname{conv}(P, v))=\frac{|\phi(v)|}{\left|\phi\left(v^{\prime}\right)\right|} \operatorname{vol}\left(\operatorname{conv}\left(P, v^{\prime}\right)\right) .
$$

Relation (7.1) admits a generalization: If $P, Q$ are a $p$-, $q$-dimensional polytopes, then the $1+p+q$-dimensional polytope $P \times Q$ defined as

$$
P \times Q=\operatorname{conv}\left(\left(0, P, 0^{q}\right),\left(1,0^{p}, Q\right)\right)
$$

has volume

$$
\operatorname{vol}(P \times Q)=\operatorname{vol}(P) \operatorname{vol}(Q) \frac{p ! q !}{(1+p+q) !} .
$$

In the following we use the notation

$$
\frac{1}{2} H_{n}=\left\{x \in\{0,1\}^{n}: \sum_{i=1}^{n} x_{i} \text { even }\right\} .
$$

for the half cube.

\subsection{The big Delone polytope}

As we shall prove later, there is only one Delone polyope of $\left[L_{n}, Q_{n}\right]$ where the maximum circumradius is attained. It is the polytope $P_{n}$ which is defined as follows. If $n$ is even then $P_{n}$ has the vertices

$$
\left((1 / 2)^{n-1}, 1\right) \pm e_{i}, i=1, \ldots, n-1, \quad\left((1 / 2)^{n-1},-1\right), \quad\left(\frac{1}{2} H_{n-1}, 0\right),
$$

If $n$ is odd, then $P_{n}$ has the vertices

$$
\left((1 / 2)^{n-1}, \pm 1\right) \pm e_{i}, i=1, \ldots, n-1, \quad\left(\frac{1}{2} H_{n-1}, 0\right) .
$$

The squared circumradius of $P_{n}$ is

$$
\mu_{P_{n}}= \begin{cases}(n-2)^{2} /(4(n-3)), & \text { if } n \text { even } \\ (n-1) / 4, & \text { if } n \text { odd. }\end{cases}
$$

The center of $P_{n}$ is

$$
c_{P_{n}}= \begin{cases}\left((1 / 2)^{n-1}, 1 /(n-3)\right), & \text { if } n \text { even, } \\ \left((1 / 2)^{n-1}, 0\right), & \text { if } n \text { odd. }\end{cases}
$$

It is proved in [16] that $P_{n}$ uniquely determines $\left[L_{n}, Q_{n}\right]$ if $n \geqslant 6$. So the quadratic function $f_{n}$ corresponding to $P_{n}$ is inhomogeneous perfect. It is also inhomogenous extreme:

LEMma 7.2. - The quadratic function $f_{n}$ is inhomogeneous eutactic. 
Proof. - The polytope $P_{n}$ has three orbits of vertices if $n$ is even which can be distinguished by considering the last coordinate: $-1,0,+1$. Then, the following coefficients satisfy the eutaxy condition

$$
\begin{aligned}
a_{-1} & =(n-2) /\left(2 n(n-3)^{2}\right), \\
a_{0} & =\left((n-2)\left(n^{2}-5 n+2\right)\right) /\left(2^{n-2} n(n-3)^{2}\right), \\
a_{1} & =2 /\left(n(n-3)^{2}\right) .
\end{aligned}
$$

The polytope $P_{n}$ has only two orbits of vertices if $n$ is odd which can be distinguished by considering the last coordinate: $\pm 1,0$. Then, the following coefficients satisfy the eutaxy condition

$$
\begin{aligned}
a_{ \pm 1} & =1 /(4 n(n-5)), \\
a_{0} & =\left(n^{2}-6 n+1\right) /\left(2^{n-2} n(n-5)\right) .
\end{aligned}
$$

The following lower bound on the volume of $P_{n}$ will turn out to be tight.

LEMma 7.3. - The volume of $P_{n}$ is at least $V_{n}$ where

$$
\begin{aligned}
V_{n}=2( & n-1) \frac{1}{n(n-1)}\left(1-\frac{2^{n-3}}{(n-2) !}\right)+2^{n-2} 2^{n-3} \frac{n-3}{n !} \\
& +\sum_{j=3}^{n-3} \frac{2^{n-2}(n-1) !}{(i+1) ! 2^{j-1} j !}\left(j !-2^{j-1}\right) \frac{n-j-1}{2 n !} \\
& +\frac{2^{n-1}}{n !}+2^{n-2} \frac{n-1}{2 n !}+2^{n-2} \frac{n-3}{2 n !}
\end{aligned}
$$

if $n$ is even, and

$$
\begin{aligned}
V_{n}=2( & n-1)(n-2) \frac{4}{n(n-1)(n-2)}\left(1-\frac{2^{n-4}}{(n-3) !}\right)+2^{n-1} \frac{n-1}{2 n !} \\
& +\sum_{j=3}^{n-4} \frac{2^{n-1}(n-1) !}{(i+1) ! 2^{j-1} j !}\left(j !-2^{j-1}\right) \frac{n-j-1}{2 n !} \\
& +2^{n-1} 2^{n-3} \frac{n-3}{n !}+2 \frac{2^{n-1}}{n !}+2^{n-2}(n-1) \frac{n-4}{n !}
\end{aligned}
$$

if $n$ is odd.

Proof. - Denote by $\mathcal{F}(P)$ the set of facets of $P$ and by $c$ the point $\left((1 / 2)^{n-1}, 0\right)$. We have

$$
\operatorname{vol}\left(P_{n}\right)=\sum_{F \in \mathcal{F}\left(P_{n}\right)} \operatorname{vol}(\operatorname{conv}(F, c)) .
$$


Since $c$ is invariant under the automorphism group of $P_{n}$, the above sum can be grouped by orbits of facets of $P_{n}$.

Below, we list the facets $F$ of $P_{n}$. The first line gives the separating hyperplane, the second line contains the list of incident vertices, the third line contains the volume $\operatorname{vol}(\operatorname{conv}(F, c))$ and the last line contains the size of the orbits. We frequently make use of the transformation $g$ defined by

$$
g\left(x_{1}, x_{2}, \ldots, x_{n}\right)=\left(1-x_{1}, x_{2}, \ldots, x_{n}\right) .
$$

- Facet $F_{1}$ : a cross polytope

$-\sum_{j=1}^{n-1} x_{j}+(n-5) / 2 x_{n} \geqslant 1$,

$-g\left(e_{j}\right), g\left(\left((1 / 2)^{n-1}, 1\right)-e_{j}\right)$ for $1 \leqslant j \leqslant n-1$,

$-2^{n-3} / n !(n-3)$,

$-2^{n-2}$.

- Facet $F_{2}$ : a cross polytope

$-x_{n} \leqslant 1$,

$-\left((1 / 2)^{n-1}, 1\right) \pm e_{j}$ for $1 \leqslant j \leqslant n-1$,

$-2^{n-1} / n !$,

-1 if $n$ even, 2 if $n$ odd.

- Facet $F_{3}$ : simplex

$-\sum_{i=1}^{n-1} x_{i}+(n-3) / 2 x_{n} \geqslant 0$,

$-0,\left((1 / 2)^{n-1}, 1\right)-e_{j}$ for $1 \leqslant j \leqslant n-1$,

$-(n-1) / 2 n !$,

$-2^{n-2}$ if $n$ even, $2^{n-1}$ if $n$ odd.

- Facet $F_{4}$ : only if $n$ even

$-2 x_{1}-x_{n} \geqslant 0$,

- $\left((1 / 2)^{n-1}, 1\right),\left(0, \frac{1}{2} H_{n-2}, 0\right)$ and $\left(-1 / 2,(1 / 2)^{n-2},-1\right)$,

$-1 / n(n-1)\left(1-2^{n-3} /(n-2) !\right)$.

$-2(n-1)$.

- Facet $F_{5}$ : simplex, only if $n$ even

$-\sum_{i=1}^{n-1} x_{i}+(n-1) / 2 x_{n} \geqslant 1$,

$-\left((1 / 2)^{n-1},-1\right), g\left(e_{j}\right)$ for $1 \leqslant j \leqslant n-1$,

$-(n-3) / 2 n !$,

$-2^{n-2}$.

- Facet $F_{6}$ : only if $n$ odd

$-x_{1}+x_{2} \geqslant 0$,

- $\left((1 / 2)^{n-1}, \pm 1\right)-e_{j}$ for $j=1,2,\left(0,0, \frac{1}{2} H_{n-3}, 0\right)$,

$-4 /(n(n-1)(n-2))\left(1-2^{n-4} /(n-3) !\right)$,

$-2(n-1)(n-2)$.

- Facet $F_{7}$ : simplex, only if $n$ odd

$-\sum_{i=1}^{n-2} x_{i}+(n-4) x_{n-1} \geqslant 1$, 
$-g\left(e_{j}\right)$ for $1 \leqslant j \leqslant n-2,\left((1 / 2)^{n-2},-1 / 2, \pm 1\right)$,

$-(n-4) / n !$

$-2^{n-2}(n-1)$.

- Facet $F_{i, j}$ : for $i+j=n-2, j \geqslant 3$ and $i \geqslant 1$ for $n$ even, $i \geqslant 2$ for $n$ odd

$-\sum_{k=j+1}^{n-1} x_{j}+(1-i) / 2 x_{n} \geqslant 0$,

$-\left(\frac{1}{2} H_{j}, 0^{i+1}, 0\right),\left((1 / 2)^{n-1}, 1\right)-e_{k}$ for $j+1 \leqslant k \leqslant n-1$,

$-\left(j !-2^{j-1}\right)(n-j-1) / 2(n !)$,

$-(i+1) ! 2^{j-1} j !$

\subsection{Proof of Theorem 7.1}

We only have to show that for every Delone polytope $P$ of $\left[L_{n}, Q_{n}\right]$ which is not equivalent to $P_{n}$ we have $\mu_{P}<\mu_{P_{n}}$.

We now construct the remaining classes of Delone polytopes of $\left[L_{n}, Q_{n}\right]$ : If $n$ is even we have one additional class and if $n$ is odd we have two additional classes.

- If $i+j=n-1$ and $3 \leqslant i \leqslant j$, we denote by $H_{i, j}$ the polytope with vertices

$$
\left(\frac{1}{2} H_{i}, 0^{j-1}, 0\right),\left((1 / 2)^{i},(1 / 2)^{j}-g\left(\frac{1}{2} H_{j}\right), 1\right) .
$$

The size of the stabilizer is

$$
\left|\operatorname{Stab}\left(H_{i, j}\right)\right|= \begin{cases}2^{i-1} i ! 2^{j-1} j !, & \text { if } i \neq j, \\ 2 \times 2^{i-1} i ! 2^{j-1} j !, & \text { if } i=j .\end{cases}
$$

Using the formula for the product polytope we get

$$
\operatorname{vol}\left(H_{i, j}\right)=\left(1-\frac{2^{i-1}}{i !}\right)\left(1-\frac{2^{j-1}}{j !}\right) \frac{i ! j !}{n !}=\left(i !-2^{i-1}\right)\left(j !-2^{j-1}\right) \frac{1}{n !} .
$$

We set $C=n-3$ if $n$ is even and $C=n-5$ if $n$ is odd. The center of $H_{i, j}$ is

$$
c_{H_{i, j}}=\left((1 / 2)^{i}, 0^{j}, \alpha\right), \text { with } \alpha=\frac{C+j-i}{2 C} .
$$

The squared radius of the sphere around $H_{i, j}$ is

$$
\mu_{H_{i, j}}=\frac{C^{2}+2 C(n-1)+(j-i)^{2}}{16 C}<\mu_{P_{n}} .
$$


- If $n$ is odd, then the simplex $S_{n}$ with vertex set

$$
0,\left(0^{n-1}, 2\right),\left((1 / 2)^{n-1}, 1\right)-e_{j}, \text { with } j=1, \ldots, n-1,
$$

is a Delone polytope. We have

$$
\begin{aligned}
\left|\operatorname{Stab}\left(S_{n}\right)\right| & =2(n-1) ! \\
\operatorname{vol}\left(S_{n}\right) & =\frac{n-3}{n !}, \\
c_{S_{n}} & =\left((1 /(n-3))^{n-1}, 1\right), \\
\mu_{S_{n}} & =\frac{n-5}{4}+\frac{n-1}{(n-3)^{2}}<\mu_{P_{n}} .
\end{aligned}
$$

Now we finish the proof by a volume computation showing that our list of orbits is complete. Denote by $O\left(D_{1}\right), \ldots, O\left(D_{r}\right)$ the orbits of Delone polytope of $\left[L_{n}, Q_{n}\right]$ of representative $D_{i}$. On the one hand, we have

$$
2=\sum_{i=1}^{r}\left|O\left(D_{i}\right)\right| \operatorname{vol}\left(D_{i}\right)
$$

On the other hand, we have the equality

$$
2=\sum_{i=1}^{\frac{n-2}{2}}\left|O\left(H_{i, j}\right)\right| \operatorname{vol}\left(H_{i, j}\right)+2 V_{n},
$$

if $n$ is even, and

$$
2=\left|O\left(S_{n}\right)\right| \operatorname{vol}\left(S_{n}\right)+\sum_{i=1}^{\frac{n-1}{2}}\left|O\left(H_{i, j}\right)\right| \operatorname{vol}\left(H_{i, j}\right)+V_{n},
$$

if $n$ is odd. This implies that $\operatorname{vol}\left(P_{n}\right)=V_{n}$ and that the list of orbits of Delone polytopes is complete. This finishes the proof of the theorem.

\section{Acknowledgements}

We thank Peter McMullen for proposing the name covering pessima. The third author thanks Rudolf Scharlau for his suggestion to work on the covering problem and Joseph Oesterlé for an interesting discussion during the DIAMANT symposium in November 2010. We thank the referee for careful reading of our manuscript and insightful comments.

We started this research during the Junior Trimester Program (February 2008-April 2008) on "Computational Mathematics". Then, part of this research was done at the Mathematisches Forschungsinstitut Oberwolfach during a stay within the Research in Pairs Programme from May 3, 2009 to May 16, 2009. We thank both institutes for their hospitality and support. 


\section{BIBLIOGRAPHY}

[1] A. Ash, "On eutactic forms", Canad. J. Math. 29 (1977), no. 5, p. 1040-1054.

[2] E. S. BArnes \& T. J. Dickson, "Extreme coverings of $n$-space by spheres", J. Austral. Math. Soc. 7 (1967), p. 115-127.

[3] A.-M. Bergé \& J. Martinet, "On weakly eutactic forms", J. Lond. Math. Soc. (2) 75 (2007), no. 1, p. 187-198.

[4] H. F. Blichfeldt, "The minimum values of positive quadratic forms in six, seven and eight variables", Math. Z. 39 (1935), no. 1, p. 1-15.

[5] S. Boyd \& L. Vandenberghe, Convex optimization, Cambridge University Press, Cambridge, 2004, xiv+716 pages.

[6] H. Cohn \& A. Kumar, "Universally optimal distribution of points on spheres", J. Amer. Math. Soc. 20 (2007), no. 1, p. 99-148.

[7] - "Optimality and uniqueness of the Leech lattice among lattices", Ann. of Math. (2) $\mathbf{1 7 0}$ (2009), no. 3, p. 1003-1050.

[8] J. H. Conway \& N. J. A. Slonne, "The cell structures of certain lattices", in Miscellanea mathematica, Springer, Berlin, 1991, p. 71-107.

[9] — Sphere packings, lattices and groups, third ed., Grundlehren der Mathematischen Wissenschaften [Fundamental Principles of Mathematical Sciences], vol. 290, Springer-Verlag, New York, 1999, With additional contributions by E. Bannai, R. E. Borcherds, J. Leech, S. P. Norton, A. M. Odlyzko, R. A. Parker, L. Queen and B. B. Venkov, lxxiv+703 pages.

[10] H. S. M. Coxeter, Regular polytopes, third ed., Dover Publications Inc., New York, 1973, xiv+321 pages.

[11] B. Delone, N. Dolbilin, S. Ryshkov \& M. Shtogrin, "A new construction in the theory of lattice coverings of an n-dimensional space by equal spheres.", Math. USSR, Izv. 4 (1970), p. 293-302.

[12] P. Delsarte, J. M. Goethals \& J. J. Seidel, "Spherical codes and designs", Geometriae Dedicata 6 (1977), no. 3, p. 363-388.

[13] M. M. Deza \& M. Laurent, Geometry of cuts and metrics, Algorithms and Combinatorics, vol. 15, Springer-Verlag, Berlin, 1997, xii+587 pages.

[14] M. Dutour Sikirić, "Polyhedral package", http://www.liga.ens.fr/ dutour/ polyhedral/.

[15] - "The six-dimensional Delaunay polytopes", European J. Combin. 25 (2004), no. 4, p. 535-548.

[16] - "Infinite series of extreme Delaunay polytope", European J. Combin. 26 (2005), p. 129-132.

[17] M. Dutour Sikirić, R. Erdahl \& K. Rybnikov, "Perfect Delaunay polytopes in low dimensions", Integers 7 (2007), p. A39, 49.

[18] M. Dutour Sikirić, A. Schürmann \& F. VAllentin, "A generalization of Voronoi's reduction theory and its application", Duke Math. J. 142 (2008), no. 1, p. $127-164$.

[19] - "Complexity and algorithms for computing Voronoi cells of lattices", Math. Comp. 78 (2009), no. 267, p. 1713-1731.

[20] R. M. ERdahl, "A cone of inhomogeneous second-order polynomials", Discrete Comput. Geom. 8 (1992), no. 4, p. 387-416. 
[21] R. M. Erdahl \& K. Rybnikov, "On Voronoi's two tilings of the cone of metrical forms", Rend. Circ. Mat. Palermo (2) Suppl. (2002), no. 70, part I, p. 279-296, IV International Conference in "Stochastic Geometry, Convex Bodies, Empirical Measures \& Applications to Engineering Science", Vol. I (Tropea, 2001).

[22] R. M. Erdahl \& S. S. Ryshkov, "The empty sphere", Canad. J. Math. 39 (1987), no. 4 , p. $794-824$.

[23] W. Lempken, B. Schröder \& P. H. Tiep, "Symmetric squares, spherical designs, and lattice minima", J. Algebra 240 (2001), no. 1, p. 185-208, With an appendix by Christine Bachoc and Tiep.

[24] J. Martinet, Perfect lattices in Euclidean spaces, Grundlehren der Mathematischen Wissenschaften [Fundamental Principles of Mathematical Sciences], vol. 327, Springer-Verlag, Berlin, 2003, xxii+523 pages.

[25] C. T. McMullen, "Minkowski's conjecture, well-rounded lattices and topological dimension", J. Amer. Math. Soc. 18 (2005), no. 3, p. 711-734 (electronic).

[26] H. Minkowski, "Diskontinuitätsbereich arithmetischer Äquivalenz", J. Reine Angew. Math. 129 (1905), p. 220-274.

[27] M. Morse, "Topologically non-degenerate functions on a compact $n$-manifold $M$.", J. Analyse Math. 7 (1959), p. 189-208.

[28] G. Nebe \& B. Venkov, "Low-dimensional strongly perfect lattices. I. The 12dimensional case", Enseign. Math. (2) 51 (2005), no. 1-2, p. 129-163.

[29] J. Notтеваum, "Sphärische 4-designs in Gittern", Universität Oldenburg, 1995.

[30] S. S. Ryshkov \& R. M. Erdahl, "The empty sphere. II", Canad. J. Math. 40 (1988), no. 5, p. 1058-1073.

[31] A. Schrijver, Theory of linear and integer programming, Wiley-Interscience Series in Discrete Mathematics, John Wiley \& Sons Ltd., Chichester, 1986, A WileyInterscience Publication, xii+471 pages.

[32] A. Schürmann, Computational geometry of positive definite quadratic forms, University Lecture Series, vol. 48, American Mathematical Society, Providence, RI, 2009, Polyhedral reduction theories, algorithms, and applications, xvi+162 pages.

[33] A. Schürmann \& F. VALlentin, "Local covering optimality of lattices: Leech lattice versus root lattice $E_{8}$ ", Int. Math. Res. Not. (2005), no. 32, p. 1937-1955.

[34] - "Computational approaches to lattice packing and covering problems", Discrete Comput. Geom. 35 (2006), no. 1, p. 73-116.

[35] M. I. ŠTogrin, "Locally quasidensest lattice packings of spheres", Dokl. Akad. Nauk SSSR 218 (1974), p. 62-65.

[36] F. Vallentin, "Sphere coverings, lattices, and tilings (in low dimensions)", $\mathrm{PhD}$ Thesis, Center for Mathematical Sciences, Munich University of Technology, 2003, http://tumb1.biblio.tu-muenchen.de/publ/diss/ma/2003/vallentin.html.

[37] B. B. Venkov, "Réseaux euclidean, designs sphériques, et formes modulaires", Monogr. Enseign. Math. 37 (2001), p. 10-86., Enseignement Math., Geneva.

[38] N. M. Vetčinkin, "Uniqueness of classes of positive quadratic forms, on which values of Hermite constants are reached for $6 \leqslant n \leqslant 8$ ", Trudy Mat. Inst. Steklov. 152 (1980), p. 34-86, 237, Geometry of positive quadratic forms.

[39] G. F. Voronor, "Nouvelles applications des paramètres continues à la théorie des formes quadratiques 1: Sur quelques propriétés des formes quadratiques positives parfaites", J. Reine Angew. Math. 133 (1908), p. 97-178. 
[40] _ - "Nouvelles applications des paramètres continus à la théorie des formes quadratiques. Deuxiéme Mémoire. Recherches sur les parallélloedres primitifs", J. Reine Angew. Math. 134 (1908), p. 198-287, and 136 (1909), 67-181.

Manuscrit reçu le 15 décembre 2010, accepté le 23 août 2011.

Mathieu DUTOUR SIKIRIĆ

Rudjer Bosković Institute

Bijenicka 54, 10000 Zagreb (Croatia)

mdsikir@irb.hr

Achill SCHÜRMANN

Universität Rostock

Institut für Mathematik

18051 Rostock (Germany)

achill.schuermann@uni-rostock.de

Frank VALLENTIN

Technical University of Delft

Delft Institute of Applied Mathematics

P.O. Box 5031, 2600 GA Delft (The Netherlands)

f.vallentin@tudelft.nl 